\title{
Differences in folate production by bifidobacteria of different origins
}

\author{
Hirosuke SUGAHARA ${ }^{1 *}$, Toshitaka ODAMAKI ${ }^{1}$, Nanami HASHIKURA ${ }^{1}$, Fumiaki ABE ${ }^{1}$ and Jin-zhong XIAO ${ }^{1}$ \\ ${ }^{1}$ Food Science and Technology Institute, Morinaga Milk Industry Co., Ltd., 5-1-83 Higashihara, Zama, Kanagawa 252-8583, Japan
}

Received March 31, 2015; Accepted July 15, 2015; Published online in J-STAGE August 5, 2015

Bifidobacteria are known to produce folate, a vital nutrient for humans. Previous studies have suggested that the ability to produce folate is strain dependent, but further adequate evaluation is needed. In this study, a total of 44 strains, including 12 species and 7 subspecies, of bifidobacteria were investigated for the production of folate during cultivation in medium containing essential levels of folate for growth of the tested strains. An in vitro assay showed that all strains of human-residential bifidobacteria (HRB) were able to produce folate, whereas most strains of non-HRB were not, with the exception of the $B$. thermophilum and $B$. longum ssp. suis strains. The differences in the in vivo production of folate by HRB and non-HRB were confirmed using mono-associated mice. The fecal folate concentrations, blood levels of hemoglobin and mean corpuscular volumes were significantly higher in the mice colonized with a folate producer, B. longum subsp. longum, compared with mice colonized with a nonproducer, B. animalis subsp. lactis. Our results confirmed the differences in folate production between HRB and non-HRB strains and suggested the benefit of HRB to hosts from the perspective of potential folate delivery.

Key words: bifidobacteria, folate, probiotic, mono-associated mice, human-residential

\section{INTRODUCTION}

Bifidobacteria naturally inhabit the gastrointestinal tract of humans and are a predominant bacterial group in the gut microbiota of infants [1]. In addition, bifidobacteria are considered to be important commensals in human-microbe interactions and are believed to play a pivotal role in maintaining a healthy gastrointestinal tract [2]. As a part of such beneficial effects, bifidobacteria are known to produce folate, which participates in many metabolic pathways, including nucleic acid and amino acid biosynthesis [3-5]. Furthermore, folate contributes to DNA methylation [6] and to the survival of regulatory T cells $[7,8]$. Therefore, folate is considered an important nutrient that participates in cell metabolism, immune development and epigenetic modification. Although dietary folate absorption occurs primarily in the duodenum and upper jejunum, the colon represents a major depot of folate produced by microbiota [9]. Therefore, folate produced by gut microbiota was considered to affect the

\footnotetext{
*Corresponding author. Mailing address: Hirosuke Sugahara, Food Science and Technology Institute, Morinaga Milk Industry Co., Ltd., 5-1-83 Higashihara, Zama, Kanagawa 252-8583, Japan. Phone: +81 46-252-3047. E-mail: h-sugahara@morinagamilk.co.jp (C)2015 BMFH Press

This is an open-access article distributed under the terms of the Creative Commons Attribution Non-Commercial No Derivatives (bync-nd) License $<$ http://creativecommons.org/licenses/by-nc-nd/3.0/>.
}

folate status of the host.

Folate production by probiotics such as lactobacilli and bifidobacteria has been previously reported [5, 9]. Pompei et al. evaluated folate production by 76 bifidobacterial strains during cultivation in a folate-free semisynthetic medium and showed that 17 strains were folate producers [10]. D'Aimmo et al. performed in vitro assays using a folate-free medium and showed that folate was produced by primate-resident bifidobacteria, such as $B$. adolescentis and $B$. dentium, but not by non-primateresident bifidobacteria [11]. These reports showed that the ability of bifidobacteria to produce folate is highly strain dependent and that species of non-human-origin bifidobacteria produce relatively lower amounts of folate compared with human-origin bifidobacteria [10-12]. However, considering that the colon represents a major depot of folate produced by microbiota [9], the folatefree media used in these studies may not accurately reflect the gut environment. D'Aimmo et al. showed that the folate-producing ability of each bifidobacterial strain was different when cultured in a synthetic folate-free or complex folate-containing medium [12]. Therefore, the potential for folate production in the gut by these strains might not have been adequately evaluated. In addition, the numbers of species studied, in particular for those of non-human origin, were limited using a medium containing essential levels of folate for growth [12].

In vivo folate production has been evaluated by 
Table 1. Species, strains and origins of bifidobacteria

\begin{tabular}{|c|c|c|c|c|c|}
\hline \multicolumn{2}{|c|}{ Human-residential bifidobacterial species } & \multirow{2}{*}{$\begin{array}{c}\text { Origin } \\
\text { Human intestine }\end{array}$} & \multicolumn{2}{|c|}{ Non-human-residential bifidobacterial species } & \multirow{2}{*}{$\begin{array}{r}\text { Origin } \\
\text { Rat feces }\end{array}$} \\
\hline B. adolescentis & ATCC $15703^{\mathrm{T}}$ & & B. animalis subsp. animalis & ATCC $25527^{\mathrm{T}}$ & \\
\hline & ATCC15704 & Human intestine & & ATCC27536 & Chicken feces \\
\hline & ATCC15706 & Human intestine & & MCC0499 & Rat feces \\
\hline B. angulatum & ATCC $27535^{\mathrm{T}}$ & Human feces & & MCC1489 & Guinea pig feces \\
\hline \multirow[t]{3}{*}{ B. bifidum } & ATCC $29521^{\mathrm{T}}$ & Human feces & B. animalis subsp. lactis & DSM10140 & Yogurt \\
\hline & ATCC15696 & Human intestine & B. longum subsp. suis & ATCC $27533^{\mathrm{T}}$ & Pig feces \\
\hline & MCC1092 & Human feces & & ATCC27532 & Pig feces \\
\hline \multirow[t]{6}{*}{ B. breve } & $\mathrm{ATCC} 15700^{\mathrm{T}}$ & Human feces & B. magnum & ATCC $27540^{\mathrm{T}}$ & Rabbit feces \\
\hline & MCC0121 & Human feces & & ATCC27681 & Rabbit feces \\
\hline & MCC0305 & Human feces & & DSM20220 & Rabbit feces \\
\hline & MCC1114 & Human feces & B. pseudolongum subsp. globosum & JCM $5820^{\mathrm{T}}$ & Cattle rumen \\
\hline & MCC1604 & Human feces & & MCC0101 & Cattle rumen \\
\hline & M-16V & Human feces & & MCC0103 & Cattle feces \\
\hline \multirow[t]{2}{*}{ B. catenulatum } & ATCC27675 & Human feces & B. pseudolongum subsp. pseudolongum & ATCC $25526^{\mathrm{T}}$ & Pig feces \\
\hline & MCC0076 & Human feces & & JCM7072 & Pig feces \\
\hline \multirow{3}{*}{ B. dentium } & DSM20436 & Human dental caries & & MCC1508 & Mouse \\
\hline & ATCC27678 & Human feces & B. thermophilum & ATCC $25525^{\mathrm{T}}$ & Pig feces \\
\hline & ATCC27679 & Human vagina & & JCM7031 & Pig feces \\
\hline \multirow[t]{2}{*}{ B. longum subsp. infantis } & ATCC $15697^{\mathrm{T}}$ & Human feces & & MCC1461 & Chicken intestine \\
\hline & JCM7009 & Human feces & & & \\
\hline \multirow[t]{4}{*}{ B. longum subsp. longum } & ATCC $15707^{\mathrm{T}}$ & Human feces & & & \\
\hline & ATCC51870 & Human feces & & & \\
\hline & MCC0300 & Human feces & & & \\
\hline & BB536 & Human feces & & & \\
\hline B. pseudocatenulatum & ATCC27919 & Human feces & & & \\
\hline
\end{tabular}

administering folate-overproducing bifidobacteria to Wistar rats with induced folate deficiency, which led to increased serum and hepatic folate levels [13]. However, because there might be other potential folate-producing bacteria in the animal microbiota [14], it is unclear whether the effect is due to direct folate production by the administered strains or via an interaction between the administered strains and the endogenous bacteria.

In this study, we investigated the in vitro folate production of 44 bifidobacterial strains belonging to 9 taxa of human-residential bifidobacteria (HRB) species and 7 taxa of non-human-residential bifidobacteria (non-HRB) species (Table 1) in a medium containing essential levels of folate, which enabled growth by all bifidobacterial strains tested and a comprehensive evaluation of bifidobacterial folate production. Furthermore, we compared the in vivo production of folate by HRB and non-HRB strains in mono-associated mice, a powerful tool for elucidating the functions of gut microbiota.

\section{MATERIALS AND METHODS}

\section{Bacterial strains}

Bifidobacterial strains were obtained from the ATCC
(American Type Culture Collection, Manassas, VA, USA), DSMZ (Deutsche Sammlung von Mikroorganismen und Zellkulturen, Braunschweig, Germany), JCM (Japan Collection of Microorganisms, Ibaraki, Japan) and MCC (Morinaga Culture Collection, Kanagawa, Japan).

\section{In vitro cultivation of bifidobacteria}

Bifidobacterium strains were subcultured in Lactobacilli MRS broth (Becton, Dickinson and Company, Sparks, MD, USA) containing $0.05 \%(\mathrm{w} / \mathrm{v})$ L-cysteine- $\mathrm{HCl}$ (MRSc broth) and anaerobically incubated at $37^{\circ} \mathrm{C}$ for $16 \mathrm{hr}$ using an Anaero Pack (Mitsubishi Gas Chemical, Tokyo, Japan). The pre-cultures were centrifuged at $10,000 \mathrm{~g}$ for 5 minutes and resuspended in fresh MRSc broth to adjust the cell numbers. A $100 \mu \mathrm{L}$ aliquot of MRSc broth containing bifidobacteria (approximately $\log 8.0$ cells) was inoculated into $3 \mathrm{~mL}$ of fresh MRSc broth and incubated anaerobically at $37^{\circ} \mathrm{C}$ for $24 \mathrm{hr}$ using an Anaero Pack. The cell number of each culture was estimated based on the optical density at $660 \mathrm{~nm}$ $\left(\mathrm{OD}_{660}\right)$ and the coefficient between the cell number and $\mathrm{OD}_{660}$ of each strain, which were determined using a bacterial counting chamber (ERMA Inc., Tokyo, Japan) under a phase-contrast microscope (BX-51; Olympus 
Corporation, Tokyo, Japan) and a microplate reader (Multiskan; Thermo Fisher Scientific Inc., Waltham, MA, USA), respectively. The culture was centrifuged, and the supernatant was filtered through a $0.22 \mu \mathrm{m}$ filter (Millex-GV; Merck Millipore, Billerica, MA, USA) and frozen at $-20^{\circ} \mathrm{C}$ until use.

\section{Folate bioassays}

A bioassay method utilizing Lactobacillus rhamnosus ATCC 7469 was employed to determine the folate concentration in the supernatant, as previously described [13] with minor modifications. Strain ATCC 7469 was cultivated at $37^{\circ} \mathrm{C}$ for $16 \mathrm{hr}$, washed three times with saline and suspended in saline as an inoculum for the assay $\left(\mathrm{OD}_{660}=0.07\right)$. Twofold concentrated Bacto Folic Acid Casei Medium (Becton, Dickinson and Company, Sparks, MD, USA) supplemented with $0.1 \%$ L-ascorbic acid was autoclaved at $121^{\circ} \mathrm{C}$ for 5 minutes. The autoclaved medium was inoculated with a $1 \%$ inoculum of strain ATCC 7469, mixed with an equal volume of each sample or standard for folate quantification, and then incubated anaerobically for 48 hours in a 96-well microplate. The 96-well microplate was vigorously shaken, and the absorbance was measured at $630 \mathrm{~nm}$.

\section{Animal study}

Germ-free BALB/c mice (10-13 weeks old, female, $\mathrm{n}=3$ ) were obtained from Sankyo Labo Service Corporation (Tokyo, Japan) and housed in gnotobiotic vinyl isolators. Sterile water and a $\gamma$-ray-sterilized AIN93G diet (Funabashi Farm, Chiba, Japan) were provided ad libitum. Two strains, B. longum subsp. longum BB536, a strain of HRB with a relatively high potential for in vitro folate production, and B. animalis spp. lactis DSM 10140, a non-HRB species with no ability for in vitro folate production, were tested in an animal study. Bifidobacteria were inoculated by administering a single dosage of drinking water containing one of the bifidobacterial strains (approximately $\log 8.3$ cells) on day 0 . Fecal and serum samples were collected on days 7, 14, 21 and 28. All animal experiments were approved by the Institutional Animal Care and Use Committee of Morinaga Milk Industry Co. Ltd., and were performed in accordance with the Guide for the Care and Use of Laboratory Animals of Morinaga Milk Industry Co., Ltd.

The fecal samples were homogenized in phosphatebuffered saline (PBS) and centrifuged at 10,000 $\mathrm{g}$ for 10 minutes. For quantification of bacterial cell numbers, the pellets were collected and frozen at $-80^{\circ} \mathrm{C}$ until use. For quantification of the folate level, the fecal supernatant and serum were filtrated through a $0.22-\mu \mathrm{m}$ filter (Millex-
GV; Merck Millipore) and subjected to folate bioassays as described above.

\section{Quantification of fecal bacterial cell numbers}

For DNA extraction, fecal pellets of $20 \mathrm{mg}$ were suspended in $450 \mu \mathrm{L}$ of extraction buffer $(100 \mathrm{mM}$ Tris-HCl and $40 \mathrm{mM}$ EDTA at $\mathrm{pH} 9.0$ ) with $50 \mu \mathrm{L}$ of $10 \%$ SDS. Glass beads (300 mg, $0.1 \mathrm{~mm}$ diameter) and $500 \mu \mathrm{L}$ of TE buffer-saturated phenol were added to the suspension, and the mixtures were vigorously shaken for $180 \mathrm{sec}$ with an MB801 Multi-beads shocker (Yasui Kikai Corporation, Osaka, Japan) device at 2,700 rpm. After centrifugation, $400 \mu \mathrm{L}$ of the supernatant was extracted with phenol/chloroform, and $250 \mu \mathrm{L}$ of the supernatant was precipitated with isopropanol. The precipitates were washed with $70 \%$ ethanol and dissolved in $200 \mu \mathrm{L}$ of Tris-EDTA buffer ( $\mathrm{pH} 8.0$ ).

Extracted bacterial DNA was subjected to realtime PCR (qPCR), as previously described [15]. For quantification of bifidobacterial cell number, the PCR primers used in this study were g-Bifid-F ( $5^{\prime}$ CTCCTGGAAACGGGTGG $3^{\prime}$ ) and g-Bifid-R (3' ACATCTATAGCCCTTCTTGTGG 5') [16]. A standard curve was prepared with $B$. breve ATCC 15700 using cell counts, which were measured using a bacterial counting chamber [17].

\section{Hematological evaluations}

The blood used for the hematology analysis was treated with tripotassium ethylenediaminetetraacetate(EDTA-3 K, Nacalai Tesque, Inc., Kyoto, Japan). The hematological examination was carried out using an automatic analyzer (Sysmex KX-21NV, Sysmex Corporation, Hyogo, Japan) to determine the red blood cell count (RBC), hemoglobin concentration (HGB), hematocrit level (HCT), and mean corpuscular volume (MCV).

\section{Statistical analyses}

Differences in folate levels in the gnotobiotic and germ-free mice were analyzed by a two-way repeatedmeasures ANOVA, with the folate level as the first factor and time as the second factor, followed by Tukey's honestly significant difference (HSD) post hoc test for analyzing sequential changes. Because the interaction effect between the time point and folate level did not show a significant difference, the intergroup folate levels were compared as the main effects. In addition, the folate levels at each time point in the gnotobiotic and germ-free mice were analyzed by a one-way ANOVA, followed by Tukey's HSD post hoc test for analyzing temporal changes. A correlation analysis between fecal and blood 
(A)

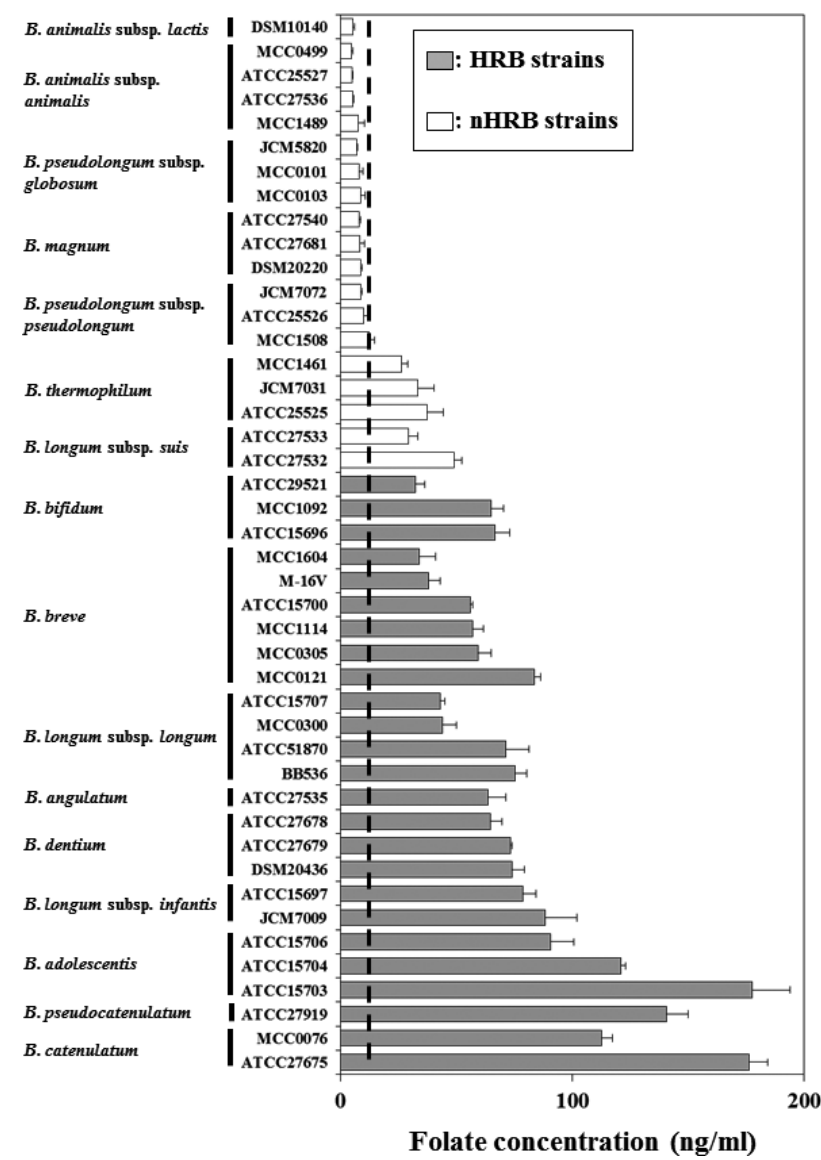

(B)

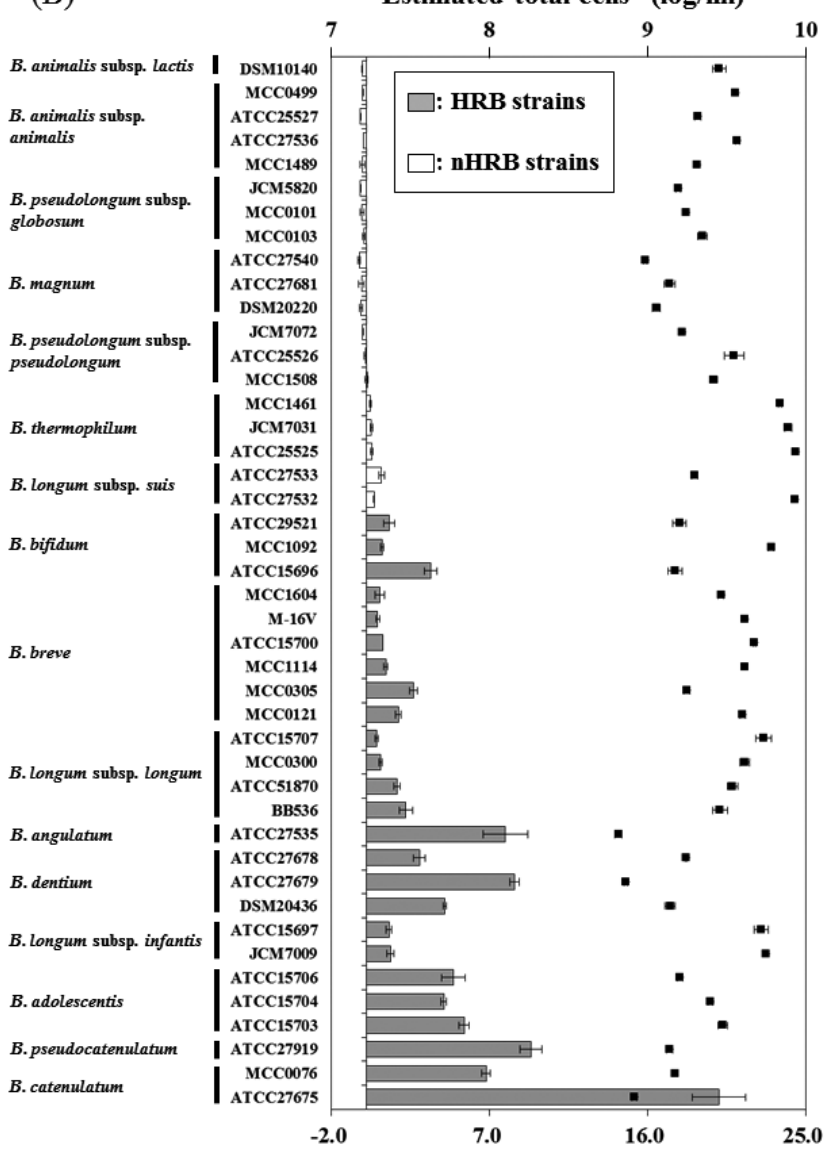

Folate production/estimated total cells (ng/108 cells)

Fig. 1. In vitro assay of folate synthesis by bifidobacterial strains

(A) Folate concentrations in culture supernatant after cultivation

Data are shown as the mean $\pm \mathrm{SD}(\mathrm{n}=3)$. Bars indicate folate concentrations. Dashed lines indicate averages of initial folate levels in MRSc broth

(B) In vitro productivity of folate with growth of bifidobacteria

Folate production was calculated by subtracting the initial folate level $(12.1 \mathrm{ng} / \mathrm{ml})$ from the folate level of the culture supernatant. Data are shown as the mean $\pm \operatorname{SD}(n=3)$. Bars indicate averages of net values of folate productivity per $10^{8}$ cells, and black squares indicate averages of estimated total cells in MRSc broth after cultivation. Initial cells were approximately log 8.0 for each strain.

folate levels was performed using Pearson's Correlation Coefficient, and inter-group difference in hematological scores were analyzed using a one-way ANOVA with Tukey's HSD post hoc test.

All analyses were performed using the IBM SPSS Statistics, version 22.0, statistical software (IBM Corp., Armonk, NY, USA). For all statements, $p$ values of $<0.05$ were considered statistically significant.

\section{RESULTS}

\section{In vitro production of folate}

The folate concentrations in the cell-free supernatants of the 44 bifidobacterial strains after cultivation in
MRSc are shown in Fig. 1A. The initial folate level in the MRSc was measured by triplicate test (mean \pm SD, $12.1 \pm 1.29 \mathrm{ng} / \mathrm{ml})$. Folate levels in the supernatants of all HRB strains were higher than the initial folate level in the medium, whereas those in the supernatants of most of the non-HRB strains were lower than the initial level in the medium, with the exception of the B. thermophilum and $B$. longum subsp. suis strains. In particular, strains of the three adult-type HRB species, $B$. adolescentis, $B$. catenulatum and $B$. pseudocatenulatum, produced high amounts of folate. The net values of folate productivity per $10^{8}$ cells were higher in strains of HRB than in strains of non-HRB, with the exception of $B$. suis ATCC 27533 (Fig. 1B). 
Animal study

In vivo folate production by bifidobacterial strains and its possible effects on hosts were evaluated using mono-associated mice. We chose B. longum subsp. longum BB536 as a representative HRB strain for the test (B. longum group) because it is frequently found in the intestines of adults and infants [18, 19], and strain BB536 showed a relatively high in vitro production of folate. We chose B. animalis subsp. lactis DSM 10140 as a representative non-HRB stain (B. lactis group) because it has been well-studied as a probiotic, and strain DSM 10140 is a folate nonproducer in vitro. Both strains colonized well and maintained more than log 9.0 cells in the feces during the study period (Table 2).

To evaluate sequential changes, we analyzed fecal folate concentrations with two-way repeated-measures ANOVA. The folate concentrations in the feces of the Bifidobacterium mono-associated mice were significantly higher than in the feces of germ-free mice (vs. the $B$. lactis group, $\mathrm{p}=0.01$; vs. the $B$. longum group, $\mathrm{p}=0.01$ ). Furthermore, the folate concentrations in the feces of the mice associated with B. longum subsp. longum BB536 were significantly higher than those of the mice associated with $B$. animalis spp. lactis DSM $10140(\mathrm{p}=0.01)$. In addition, one-way ANOVA indicated significant differences in the folate concentrations among the study group; that is, there were significantly higher levels in the $B$. longum group compared with germ-free mice throughout the study period, significantly higher levels in the $B$. lactis group compared with germ-free mice at day 28 , and significantly higher levels in the $B$. longum group compared with the $B$. lactis group at day 21 (Fig. 2A). There was no significant difference in the serum folate levels among the three groups (Fig. 2B), although there was a positive correlation between the fecal and serum levels of folate $(r=0.52, p=0.01)$.

Folate has been known to contribute to some blood parameters related to erythrocytes $[3,20]$. We observed significantly higher levels of hemoglobin and larger mean corpuscular volumes in the $B$. longum group than in the $B$. lactis group (Fig. 3).

\section{DISCUSSION}

Recent genomics work has revealed that all type strains of bifidobacterial taxa isolated from humans (except for B. gallicum) or other primates (except for B. biavatii) possess the set of genes involved in tetrahydrofolate biosynthesis [21]. In addition, type strains of $B$. longum subsp. suis and B. thermophilum [21] possess the same gene homologs, whereas the type strains of the other
Table 2. Fecal bacterial cell numbers in mono-associated mice

\begin{tabular}{lcccc}
\hline \multirow{2}{*}{ Group } & \multicolumn{4}{c}{ Fecal cell numbers $\left(\log \mathrm{g}^{-1}\right)$} \\
\cline { 2 - 5 } & Day 7 & Day 14 & Day 21 & Day 28 \\
\hline B. lactis & $9.43 \pm 0.13$ & $9.35 \pm 0.10$ & $9.47 \pm 0.13$ & $9.44 \pm 0.10$ \\
B. longum & $9.46 \pm 0.02$ & $9.24 \pm 0.15$ & $9.14 \pm 0.18$ & $9.06 \pm 0.05$ \\
\hline
\end{tabular}

Data are shown as the mean $\pm \mathrm{SD}$.

non-HRB species used in our study do not. This genomic information is in accordance with our phenotypic results of in vitro folate production. D'Aimmo et al. reported folate production by all primate-residential bifidobacteria, such as $B$. adolescentis and $B$. dentium, but not by non-primate-residential bifidobacteria, such as $B$. pseudolongum subsp. globosum, B. indicum, B. asteroids and B. coryneforme [11]. However, our results showed that some strains belonging to non-primate species, such as $B$. thermophilum and B. longum subsp. suis, which were isolated from pig feces or the chicken intestine (Table 1), are also folate producers. The difference in findings may be the result of the different strains used in our study and previous studies. B. longum subsp. suis belongs to the same species of $B$. longum subsp. longum and $B$. longum subsp. infantis, which are among the dominant bifidobacterial species in both adults and infants $[18,19]$. Von Ah et al. reported the isolation of one strain of $B$. thermophilum from human infant feces [22]; this species might possess some characteristics in common with HRB. These characteristics might relate to its exception as a folate producer.

In contrast to the report by Pompei et al. [10], which showed that 41 of 57 HRB strains could neither grow nor produce folate in folate-deficient medium, we found that all of the HRB and some of the non-HRB strains could produce folate in medium containing essential levels of folate for growth of the tested strains. Although the set of strains subjected to in vitro assays were different between our study and previous studies, there were inconsistent outcomes even among some common strains such as $B$. adolescentis ATCC 15703 and B. longum subsp. longum ATCC15707. These results suggest the importance of adequate methods for the evaluation of folate production by bifidobacterial strains. Our animal study demonstrated the possibility of using administered bifidobacterial strains for direct production of folate or its derivatives without interactions with other members of the gut microbiota. Our results also showed the superiority of in vivo folate production by HRB strains compared with non-HRB strains using mono-associated mice. However, we did not expect that the folate concentration in the feces 

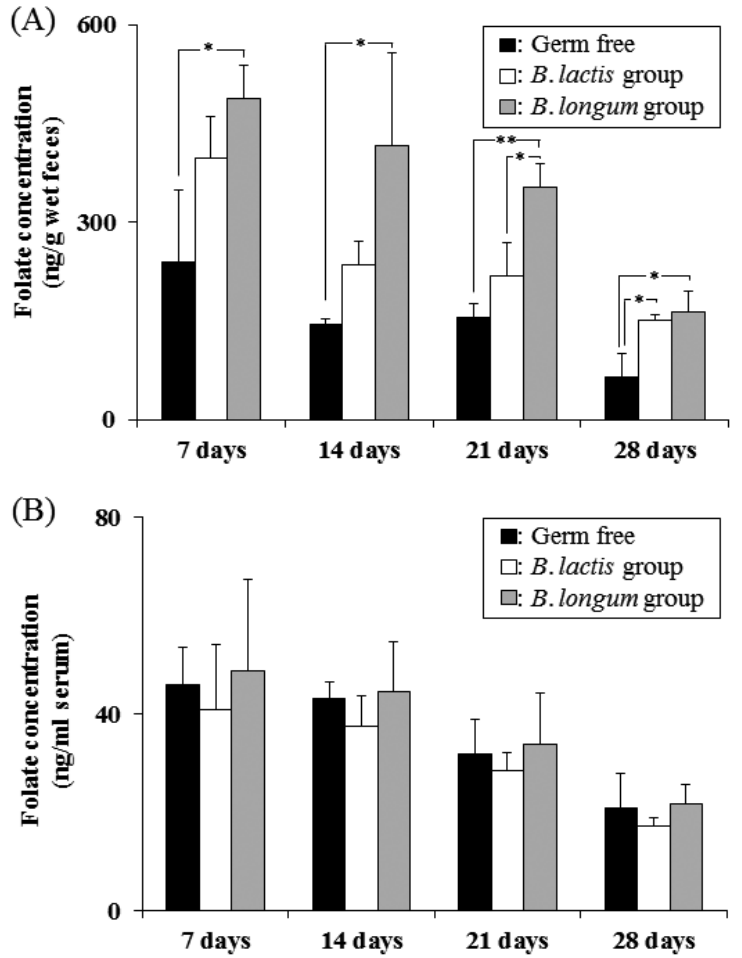

Fig. 2. Folate concentrations in germ-free and monoassociated mice

(A) Folate concentrations in feces and (B) serum. Data are shown as the mean $\pm \mathrm{SD}(\mathrm{n}=3)$. P-values were calculated using one-way ANOVA followed by Tukey's test. $* \mathrm{p}<0.05 ; * * \mathrm{p}<0.01$.

of the $B$. lactis group would be significantly higher than that of the germ-free mice, although genomic and in vitro analyses have demonstrated the lack of de novo folate production by $B$. animalis subsp. lactis DSM 10140. One possible reason for this is that folate is synthesized from intermediates such as dihydropteroate in the intestinal environment; this will be examined in a future study.

A previous study demonstrated that administration of folate-producing bifidobacteria increased the serum and hepatic folate concentrations in Wistar rats fed a lowfolate diet, suggesting that the folate status of the blood is affected by gastrointestinal folate levels [13]. The reason for the absence of a significant difference in the serum folate levels in this study is unclear; however, the folate contained in the diet $(420 \mathrm{ng} / \mathrm{g}$ as evaluated by the method described in the Materials and Methods section) might have been enough to maintain the level of serum folate as a homeostatic mechanism.

Folate is known to be a nutrient related to hemoglobin production because it functions as a carbon carrier in the formation of heme, which is the iron-containing
(A)

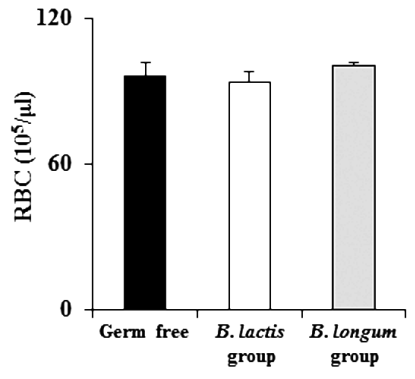

(C)

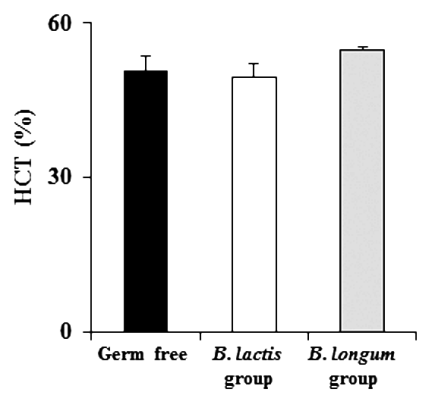

(B)

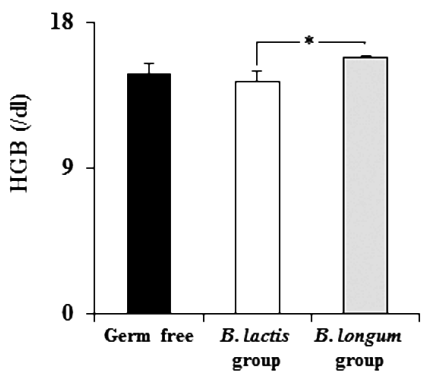

(D)

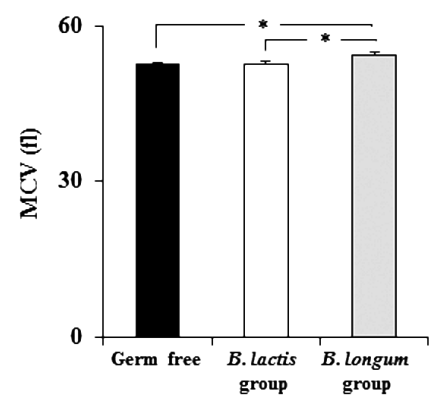

Fig. 3. Hematological evaluation of germ-free and mono-associated mice

(A) Red blood cell count (RBC), (B) hemoglobin concentration (HGB), (C) hematocrit level (HCT), and (D) mean corpuscular volume (MCV).

Data are shown as the mean $\pm \mathrm{SD}(\mathrm{n}=3)$. P-values were calculated using one-way ANOVA followed by Tukey's test. * $\mathrm{p}<0.05$.

nonprotein portion of hemoglobin [3]. We observed significantly higher levels of hemoglobin and a larger mean corpuscular volume in the $B$. longum group than in the $B$. lactis group. This result indicates that the level of folate produced by Bifidobacterium might affect hemoglobin levels. However, there was no significant difference in the hemoglobin levels between the $B$. longum group and the germ-free mice, although the fecal folate levels were significantly higher in the $B$. longum group compared with those of the germ-free mice. The reason for this is unclear, however, as hemoglobin levels can be influenced by other nutrition parameters, such as vitamin B12 and iron [23]; further analysis is needed to understand the contribution of folate to blood hemoglobin levels.

In conclusion, we demonstrated that in vitro folate production by HRB species was generally higher than that of non-HRB species in a medium containing essential levels of folate for growth. A study using monoassociated mice confirmed the direct production of folate by bifidobacterial strains in vivo. In addition, the blood 
levels of hemoglobin and the mean corpuscular volume were significantly enhanced in the mice colonized with an HRB strain compared with in the mice colonized with a non-HRB strain. Our findings suggest the utility of HRB from the perspective of potential folate delivery to the host.

\section{REFERENCES}

1. Bottacini F, Ventura M, van Sinderen D, O'Connell Motherway M. 2014. Diversity, ecology and intestinal function of bifidobacteria. Microb Cell Fact 13 Suppl 1: S4. [Medline] [CrossRef]

2. Leahy SC, Higgins DG, Fitzgerald GF, van Sinderen D. 2005. Getting better with bifidobacteria. J Appl Microbiol 98: 1303-1315. [Medline] [CrossRef]

3. Iyer R, Tomar SK. 2009. Folate: a functional food constituent. J Food Sci 74: R114-R122. [Medline] [CrossRef]

4. LeBlanc JG, Laiño JE, del Valle MJ, Vannini V, van Sinderen D, Taranto MP, de Valdez GF, de Giori GS, Sesma F. 2011. B-group vitamin production by lactic acid bacteria - current knowledge and potential applications. J Appl Microbiol 111: 1297-1309. [Medline] [CrossRef]

5. LeBlanc JG, Milani C, de Giori GS, Sesma F, van Sinderen D, Ventura M. 2013. Bacteria as vitamin suppliers to their host: a gut microbiota perspective. Curr Opin Biotechnol 24: 160-168. [Medline] [CrossRef]

6. Mischke M, Plösch T. 2013. More than just a gut instinct-the potential interplay between a baby's nutrition, its gut microbiome, and the epigenome. Am J Physiol Regul Integr Comp Physiol 304: R1065R1069. [Medline] [CrossRef]

7. Kunisawa J, Hashimoto E, Ishikawa I, Kiyono H. 2012. A pivotal role of vitamin $\mathrm{B} 9$ in the maintenance of regulatory $\mathrm{T}$ cells in vitro and in vivo. PLoS ONE 7: e32094. [Medline] [CrossRef]

8. Kinoshita M, Kayama H, Kusu T, Yamaguchi T, Kunisawa J, Kiyono H, Sakaguchi S, Takeda K. 2012. Dietary folic acid promotes survival of Foxp3+ regulatory T cells in the colon. J Immunol 189: 28692878. [Medline] [CrossRef]

9. Rossi M, Amaretti A, Raimondi S. 2011. Folate production by probiotic bacteria. Nutrients 3: 118-134. [Medline] [CrossRef]

10. Pompei A, Cordisco L, Amaretti A, Zanoni S, Matteuzzi D, Rossi M. 2007. Folate production by bifidobacteria as a potential probiotic property. Appl Environ Microbiol 73: 179-185. [Medline] [CrossRef]

11. D'Aimmo MR, Modesto M, Mattarelli P, Biavati B, Andlid T. 2014. Biosynthesis and cellular content of folate in bifidobacteria across host species with different diets. Anaerobe 30: 169-177. [Medline] [CrossRef]

12. D'Aimmo MR, Mattarelli P, Biavati B, Carlsson NG, Andlid T. 2012. The potential of bifidobacteria as a source of natural folate. J Appl Microbiol 112: 975-984. [Medline] [CrossRef]

13. Pompei A, Cordisco L, Amaretti A, Zanoni S, Raimondi S, Matteuzzi D, Rossi M. 2007. Administration of folate-producing bifidobacteria enhances folate status in Wistar rats. J Nutr 137: 2742-2746. [Medline]

14. Klipstein FA, Samloff IM. 1966. Folate synthesis by intestinal bacteria. Am J Clin Nutr 19: 237-246. [Medline]

15. Odamaki T, Sugahara H, Yonezawa S, Yaeshima $\mathrm{T}$, Iwatsuki K, Tanabe S, Tominaga T, Togashi H, Benno Y, Xiao JZ. 2012. Effect of the oral intake of yogurt containing Bifidobacterium longum BB536 on the cell numbers of enterotoxigenic Bacteroides fragilis in microbiota. Anaerobe 18: 14-18. [Medline] [CrossRef]

16. Matsuki T, Watanabe K, Fujimoto J, Kado Y, Takada T, Matsumoto K, Tanaka R. 2004. Quantitative PCR with 16S rRNA-gene-targeted species-specific primers for analysis of human intestinal bifidobacteria. Appl Environ Microbiol 70: 167-173. [Medline] [CrossRef]

17. Satoh T, Odamaki T, Namura M, Shimizu T, Iwatsuki K, Nishimoto M, Kitaoka M, Xiao JZ. 2013. In vitro comparative evaluation of the impact of lacto-N-biose I, a major building block of human milk oligosaccharides, on the fecal microbiota of infants. Anaerobe 19: 50-57. [Medline] [CrossRef]

18. Schloissnig S, Arumugam M, Sunagawa S, Mitreva M, Tap J, Zhu A, Waller A, Mende DR, Kultima JR, Martin J, Kota K, Sunyaev SR, Weinstock GM, Bork P. 2013. Genomic variation landscape of the human gut microbiome. Nature 493: 45-50. [Medline] [CrossRef]

19. Tsuji H, Oozeer R, Matsuda K, Matsuki T, Ohta T, Nomoto K, Tanaka R, Kawashima M, Kawashima K, Nagata S, Yamashiro Y. 2012. Molecular monitoring of the development of intestinal microbiota in Japanese infants. Benef Microbes 3: 113-125. [Medline] [CrossRef]

20. Fishman SM, Christian P, West KP. 2000. The role of vitamins in the prevention and control of anaemia. Public Health Nutr 3: 125-150. [Medline] [CrossRef]

21. Milani C, Lugli GA, Duranti S, Turroni F, Bottacini F, Mangifesta M, Sanchez B, Viappiani A, Mancabelli L, Taminiau B, Delcenserie V, Barrangou R, Margolles A, van Sinderen D, Ventura M. 2014. Genomic encyclopedia of type strains of the genus Bifidobacterium. Appl Environ Microbiol 80: 62906302. [Medline] [CrossRef]

22. von Ah U, Mozzetti V, Lacroix C, Kheadr EE, Fliss I, Meile L. 2007. Classification of a moderately oxygentolerant isolate from baby faeces as Bifidobacterium thermophilum. BMC Microbiol 7: 79. [Medline] [CrossRef]

23. Koury MJ, Ponka P. 2004. New insights into erythropoiesis: the roles of folate, vitamin B12, and iron. Annu Rev Nutr 24: 105-131. [Medline] [CrossRef] 